\section{Activation and Inhibition of Adrenaline Metabolism}

MANY drugs enhance or block the actions of adrenaline; but little is known as to whether they act by modifying the metabolism of the catecholamine. In this work the effects of a variety of drugs on the rate of disappearance of radioactive adrenaline in the whole mouse were examined. Details of drug dosage are given in Table 1. Mice were treated with drug and

Table 1. ACtivazion and Inhibition of Adrenatise MLtabotigm

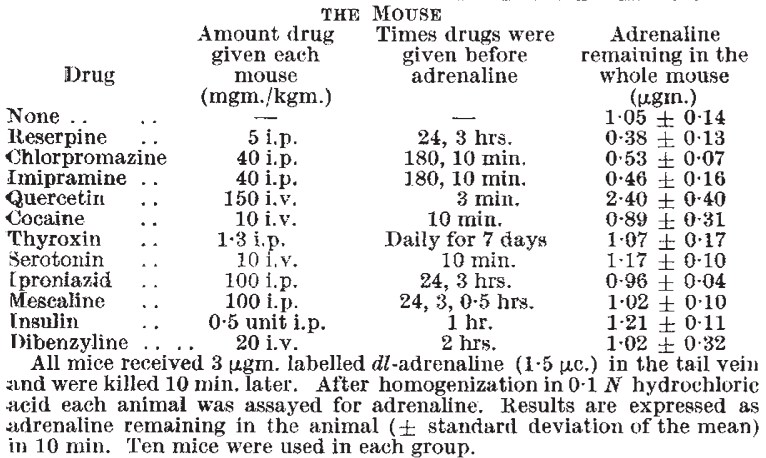

then adrenaline (3 $\mu \mathrm{gm}$.) labelled with $\mathrm{H}-3$ was administered intravenously. Ten minutes after the adrenaline injection the animals were killed, homogenized in a Waring blendor, and an aliquot of the homogenate assayed for labelled adrenaline remaining ${ }^{1}$. Control animals, untreated with drug, were given adrenaline labelled with $\mathrm{H}-3$ at a similar time.

The results of these experiments are shown in Table 1. About one-third of the administered adrenaline was found unchanged in control mice in $10 \mathrm{~min}$. The psychotropic drugs, reserpine, chlorpromazine, and imipramine ('Tofrānil') were found to increase the rate of adrenaline metabolism markedly. Quercetin, on the other hand, inhibited the disappearance of adrenaline while cocaine, dibenzyline, thyroxin, insulin, iproniazid (a monoamine oxidase inhibitor) serotonin, and mescaline had no effect.

We have previously shown that adrenaline disappears from the body in two phases ${ }^{1}$. In the first phase the amine is rapidly metabolized by $\mathrm{O}$-methylation. In the second, the disappearance of the hormone is much slower indicating that a portion of the catecholamine is bound and then slowly released and metabolized. Since reserpine has been shown to release catecholamines from binding sites ${ }^{2}$, it is presumably preventing the binding of free adrenaline. Thus, in animals treated with reserpine, adrenaline is exposed to enzymatic attack and consequently metabolized more rapidly. At present the mechanism whereby chlorpromazine and imipramine increases the destruction of adrenaline is not clear. They do not activate $\mathrm{O}$-methyl transferase, the enzyme mainly concerned with the inactivation of adrenaline ${ }^{3,4}$. Chlorpromazine and imipramine might act by prevent ing the binding of adrenaline or they might speed the transfer of the catecholamine across membrane barriers to the site of enzymatic transformation. It is possible that the central effects of these drugs are mediated by their action on adrenaline metabolism.

The catechol flavanoid, quercetin, effectively blocks the metabolism of adrenaline in vivo (Table 1). It has been previously shown that pyrogallol inhibits catechol-O-methyl transferase in vitro ${ }^{5}$ and in vivo ${ }^{4}$ At equimolar concentrations quercetin also inhibits the $\mathrm{O}$-methylation of adrenaline $i n$ vitro about 50 per cent. This inhibition appears to be competitive since it can be reversed by increasing the concentration of adrenaline. Quercetin and other flavanoids have been shown to prolong the actions of adrenaline ${ }^{6}$. In the light of the findings reported in this communication, the potentiating action on adrenaline by flavanoids is most probably due to their inhibition of catechol-O-methyl transferase.

\section{Julius Axelrod ROBERT 'TOMCHICK}

Laboratory of Clinical Science,

National Institute of Mental Health,

National Institutes of Health, Bethesda, Maryland. Sept. 21

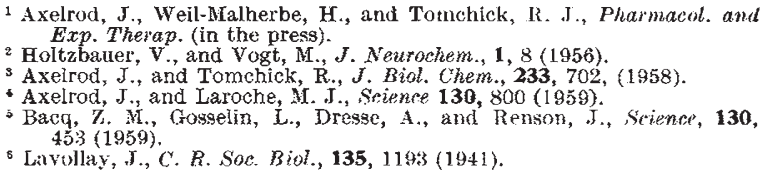

\section{Significance of the Time Relations of Humorally Co-ordinated Chromatic Responses}

In the course of investigations into the nature of the chromactivating hormones of the teleost pituitary I have had occasion to examine in detail the well-known 'bihumoral hypothesis' first put forward by Hogben and Slome ${ }^{1}$, and afterwards adopted by a number of other workers. This hypothesis seeks to show that the observed time relations of humorally co-ordinated changes in colour are incompatible with a co-ordinating mechanism based on fluctuations in the level of a single hormone.

Hogben ${ }^{2}$ and Hogben and Landgrebe ${ }^{3}$ have shown that the chromatic responses of lower vertebrates are co-ordinated largely by the eye; they found that the retina was divisible into two regions, one concerned with the white background response ( $W$ region) and the other with the black background response ( $B$ region). Illumination of the $B$ region alone resulted in melanophore-dispersion, the black background response; illumination of the $B$ and $W$ regions together resulted in melanophore-aggregation, the white background response. In the dark the melanophores assumed an intermediate condition. Experiments with hypophysectomized animals show that the pituitary is the source of hormone controlling chromatic responses; presumably the release of hormone from the gland is regulated by the appropriate regions of the retina.

Hogben and Slome ${ }^{1,4}$ observed the time relations of colour change of Xenopus laevis in response to changes in background and illumination. They found that the interval $b T d$ (notation devised by Hogben; $b T d=$ time taken to pass from equilibrium on a black background in light to equilibrium in darkness; $b T w=$ time taken to pass from equilibrium on a black background in light to equilibrium on a white backgound in light, etc.) was greater than $b T w$. Similarly, $w T d$ was greater than $w T b$. It was argued that, if a single hormone were involved in these changes, transition from a black to a white background in light or vice versa must involve the release or destruction of more hormone than transition from black or white illuminated backgrounds to darkness 'and must necessarily take longer" 4 . Since this was not so, more than one hormone must be involved in the coordination of the observed responses. Similar argu- 